\title{
Analysis of uropathogens and antibiotic suscesbility patterns in Erbil Teaching Hospital, Erbil city: A Retrospective study for one year
}

Nasik Shawkat Dogramachi ${ }^{1}$, Niyan Inaam Muhammed Yousif ${ }^{2}$, Mahmod kamal Nuri ${ }^{3}$

1 Necip Fazil Hospital, Turkey

2 College of Medicine, Hawler Medical University

3 Hawler Teaching Hospital, Erbil city

Corresponding Author: Niyan Inaam Muhammed Yousif, Niyan.innam@gmail.com

\section{ABSTRACT}

Background and objectives: The antibiotic susceptibility profile of urinary tract pathogens has frequently been changing due to the constant development of new resistance mechanisms. This study analyzed the antimicrobial susceptibility profile from patients with urinary tract infection who attended Erbil teaching hospital.

Method: A retrospective analysis of the 541 urine isolates over one year (January 2015 to December 2015) was performed at the Department of Microbiology, Erbil Teaching Hospital. Suitable bacterial isolates were identified using commercial identification methods and their MICs (minimum inhibitory concentration) were determined by automated susceptibility testing with the use of Conventional identification (ID)/(AST) antibiotic susceptibility test combination Microscan (Dade Behring), according to the manufacturers' instructions.

Results: A total of 541 urine culture sensitivity reports were analyzed 158 samples (29. 2\%) yielded significant bacteriuria; 330 samples (61\%) showed no growth, and 53 samples $(9.8 \%)$ showed mixed growth. Out of 158 positive samples (68. 4\%) were Gram-negative, (31. 6\%) were Gram-positive isolates. Escherichia coli (44. 2\%) was the commonest pathogen isolated from samples, followed by Klebsiella pneumonia (13.5\%) and Staphylococcus aureus (11.5\%). The highest sensitivity rates for E. coli and K. pneumonia were for imipenem (87\%, $85.7 \%)$, amikacin $(79.7 \%, 81 \%)$, colistin $(82.6 \%, 76.2 \%)$, respectively, while linezolid, daptomycin, Synercid were the most effective antibiotic against S.aureus.

Conclusion: E.coli, K.pneumoniae, S.aureus were the commonly isolated bacteria from urine samples in our regional teaching hospital. İmipenem and amikacin were the most effective antibiotics against E.coli, and K. pneumoniae while, daptomisin, linezolid, and synercid were the most effective antibiotics against S.aureus.

Keywords: Retrospective study, Uropathogens, Antibiotic Susceptibility. 


\section{Proceeding of the $2^{\text {nd }}$ Scientific Conference on Women's Health \\ 2-3 September 2020 - Hawler Medical University}

\section{INTRODUCTION}

Urinary tract infection (UTI) regards as one of the most common diseases experienced in the medical practice today, occurring from the neonate to the geriatric age group. ${ }^{1}$ The predominance of Enterobacteriaceae and particularly E. coli stay the main pathogen causing UTI, accounting for 75-90\% of all UTIs in both inpatients and outpatients. ${ }^{2}$ Also, Klebsiella spp., Staphylococcus spp., Proteus spp., Pseudomonas spp. and Enterococcus spp. were more often isolated from inpatients. ${ }^{3}$ A variety of antibiotics is available for treating UTIs, but changing antibiotic sensitivities make appropriate empiric treatment a moving target over time. ${ }^{4}$ Empiric antibiotic therapy is usually based on epidemiological data that are updated and adapted geographically, highlighting the importance of local and regular monitoring of bacterial resistance. ${ }^{5}$

Over the past several decades, antibiotic-resistant of Enterobacteriaceae have been increased significantly. ${ }^{6}$ E. coli and K. pneumoniae, are the most common pathogens associated with drug resistance and can exhibit resistance to multiple antibiotics ${ }^{7}$ In Western Countries, E. coli is resistant to Fluoroquinolones in $8 \%-48 \%$ and to third-generation Cephalosporin's in 3$43 \%$ of the cases. Similar to E. coli, K. pneumonia acquires resistance to multiple antibacterial drugs mainly through horizontal transfer of mobile genetic elements such as transposons or plasmids. Klebsiella is an emerging problem because the ESBL-positive (Extended Spectrum Beta-Lactamase) strains are resistant to all extended beta-lactam antibacterial drugs such as Cephalosporins. Although the treatment choice for these strains is Carbapenems, $K$. pneumonia is also the primary trigger of infections caused by Carbapenemresistant bacteria worldwide $(0-68 \%){ }^{8}$

The SA is a nosocomial bacterial pathogen and is a relatively uncommon cause of UTI, resulting in up $7 \%$ of such cases. ${ }^{9}$ Bacteriuria with SA can occur as a result of ascending infection precipitated by urinary catheterization or from a hematogenous route of infection, often resulting from intravascular device exposure, and may represent an undetected bacteremia. ${ }^{10-11}$ The SA is an opportunistic pathogen affecting both immune-competent and immunocompromised individuals, frequently resulting in significant morbidity. Many strains of SA carry a wide variety of multidrug-resistant genes on plasmids, which aid the spread of resistance among species. ${ }^{12}$ Over the past 20 years, Meticillin Resistant Staphylococcus aureus (MRSA) has emerged as an important cause of nosocomial bacteremia, and there has been a significant increase in the incidence of MRSA infections. ${ }^{13}$ Most urinary tract infections caused by MRSA are hospital aquired (HA-MRSA )infections. Generally, these 


\section{Proceeding of the $2^{\text {nd }}$ Scientific Conference on Women's Health \\ 2-3 September 2020 - Hawler Medical University}

patients are asymptomatic, but in the case of a weakened general condition, asymptomatic MRSA infection can detoriat the patient's status considerably and require treatment. ${ }^{14}$ In contrast to HA-MRSA, community-acquired (CA-MRSA) urinary tract infections offer clinical symptoms such as dysuria and pollakiuria. Of the $5.7 \%$ of the study population with CA-MRSA, no MRSA source was found other than the urinary sample. Possible reasons for the increase in community-acquired infections are the lateral dissemination of MRSA from the hospital to the community from discharged patients diagnosed with MRSA, discontinuation of therapy, and missing follow-ups. Many strains of MRSA are frequently resistant to multiple antibiotics. ${ }^{15}$ These strain carries a uniquely effective antibiotic resistance mechanism that can protect the microorganisms against all members of $\beta$-lactam antibiotics. It makes infections caused by these pathogens challenging to manage and costly to treat. ${ }^{16-17}$

The distribution of uropathogens and their susceptibility pattern to antibiotics vary locally. Therefore, knowing the frequency of the causative microorganisms and their susceptibility to various antibiotics is important ${ }^{10}$. This study was undertaken to determine the prevalence of local uropathogens in teaching hospitals in Erbil city and their antibiotic sensitivity pattern to commonly used antibiotics to provide a database.

\section{MATERIALS AND METHODS}

\section{Study Design:}

A retrospective analysis of one year (January 2015 to December 2015) was dune on the patients symptomatic of urinary tract infection that either attended medical outpatient-clinics or were admitted in Hawler Teaching Hospital's medical wards in Erbil City during that period. All the patients symptomatic of UTI were included irrespective of their comorbidities.

\section{Data collection}

All patient data were collected from the Microbiology Department records from January to December 2015 were included in the study. Similarly, all the information, including culture results and antibiotic sensitivity for isolated pathogens were collected from the records of the Microbiology Department for the study period.

\section{Laboratory diagnostic methods}

The present study was carried out in the Bacteriology Laboratory of the Department of Microbiology of Hawler Teaching Hospital. The records data showed urine samples from 


\section{Proceeding of the $2{ }^{\text {nd }}$ Scientific Conference on Women's Health 2-3 September 2020 - Hawler Medical University}

various outpatient departments (OPDs) and inpatient departments (IPDs) of the teaching hospital in Erbil city. Ethical clearance was not needed as the study was on routine laboratory isolates. The clean-catch, midstream urine samples were gathered in sterile universal containers. Urine samples were handled within two h of collection, and, in case of lag, the samples were refrigerated at $2-8^{\circ} \mathrm{C}$ for up to $6 \mathrm{~h}$. The samples were plated on Blood Agar and MacConkey Agar media by the semi-quantitative plating method. Plates were incubated aerobically overnight at $37^{\circ} \mathrm{C}$. A positive urine culture was defined as the growth of $>10$ and any colony-forming unit $[\mathrm{CFU}] / \mathrm{ml}$ of a single uropathogen for specimens obtained by cleancatch midstream, catheterization, and suprapubic methods, respectively. ${ }^{18}$ Relevant bacterial isolates were identified using commercial identification methods, and their MICs (minimum inhibitory concentration) were determined by automated susceptibility testing with use of Conventional ID/AST combination Microscan (Dade Behring), according to the manufacturers' instructions. The MIC breakpoints and quality-control protocols were based on standards of the Clinical Laboratory Standards Institute ${ }^{19}$.

\section{Ethical consideration}

Ethically, the study was cleared, and an official letter was written from (HMU) Faculty of Medicine, Department of Microbiology, and Teaching Hospital. We agreed and signed the appropriate form. Given that it is a retrospective study, informed consent was not asked. The study didn't require the patient's name or personal identification details, thereby assuring the confidentiality of the patients' information and privacy.

\section{RESULTS}

Distribution of culture results according to gender. A total of 541urine samples were analyzed for culture and sensitivity from January to December 2015. Among these cultures, 158 samples (29.2\%) yielded significant bacteriuria, 330 samples (61\%) showed no growth, and 53 samples (9.8\%) showed contamination. Among relevant positive culture 107 (30.7\%) urine cultures were from female patients, and the rest of the $51(26.4 \%)$ were from male patients, as shown in table 1. 
Proceeding of the $2^{\text {nd }}$ Scientific Conference on Women's Health 2-3 September 2020 - Hawler Medical University

Table1. Distribution of culture result according to the gender

\section{Gender}

\begin{tabular}{lcccc} 
& \multicolumn{2}{c}{ Culture result } & Total \\
\hline Nemale & $198(56.9 \%)$ & $107(30.7 \%)$ & $43(12.4 \%)$ & $348(100 \%)$ \\
\hline Male & $132(68.4 \%)$ & $51(26.4 \%)$ & $10(5.2 \%)$ & $193(100 \%)$ \\
\hline Total & $330(61 \%)$ & $158(29.2 \%)$ & $53(9.8 \%)$ & $541(100 \%)$
\end{tabular}

Prevalence and distribution of pathogens from urine: Out of 158 positive samples, (31.6\%) were Gram-positive isolates, and (68.4\%) were Gram-negative isolates. Out of them, 69 were $E$. coli (44.2\%), 21 were $K$. pneumonia (13.5\%), 18 were $S$. aureus (11.5\%), 14 were Enterococcus spp. (7 \%), 8 were S.epidermidis(5.1\%), 7 were Pseudomonas spp. (4.5\%), 6 were S. agalactiae (3.8\%), 5 were P. mirabilis (3.2\%), 3 Acinetobacter spp. (1.9\%) while other Gram-negatives were found in small numbers, as shown in table 2. The most frequently isolated organism was E. coli (43.7\%) following by K.pneumoniae (13.5\%) and S.aureus $(11.4 \%)$.

Table (2): Prevalence and distribution of pathogens from urine

\begin{tabular}{ll} 
Organism type & $\mathbf{n}(\%)$ \\
\hline Escherichia coli & $69(44.2)$ \\
Klebsiella pneumonia & $21(13.5)$ \\
Staphylococcus aureus & $18(11.5)$ \\
Enterococcus spp. & $14(9)$ \\
Staphylococcus epidermidis & $8(5.1)$ \\
Pseudomonas spp & $7(4.5)$ \\
Streptococcus agalactiae & $6(3.8)$ \\
Proteus mirabilis & $5(3.2)$ \\
Acintobacterspp & $3(1.9)$ \\
Alicaligenes spp & $1(0.6)$ \\
Others & $4(2.4)$ \\
\hline Total & $157(100)$
\end{tabular}




\section{Proceeding of the $2^{\text {nd }}$ Scientific Conference on Women's Health 2-3 September 2020 - Hawler Medical University}

\section{Antibiotic susceptibility profile of E.coli and $K$. pneuomonia samples:}

The susceptibility profile of E. coli, K. pneuomonia against various antimicrobials is shown in Figure 1. The sensitivity of E.coli as the main causative agent of UTI was highest against Imipenem, Ertapenem, colistin, amikacin, fosfomisin, nitrofurantoin and piperacillin tazobactam. $87 \%, 82.6 \%, 79.7 \%, 79.9 \%, 70.5 \%$ and $65.2 \%$ respectively. On the other hand, klebsiella strains showed high sensitivity against imipenem, amikacin, ertapenem, colistin, and levofloxacin, $85.7 \%, 81 \%, 76.2 \%, 76.2 \%, 71.8 \%$, respectively.

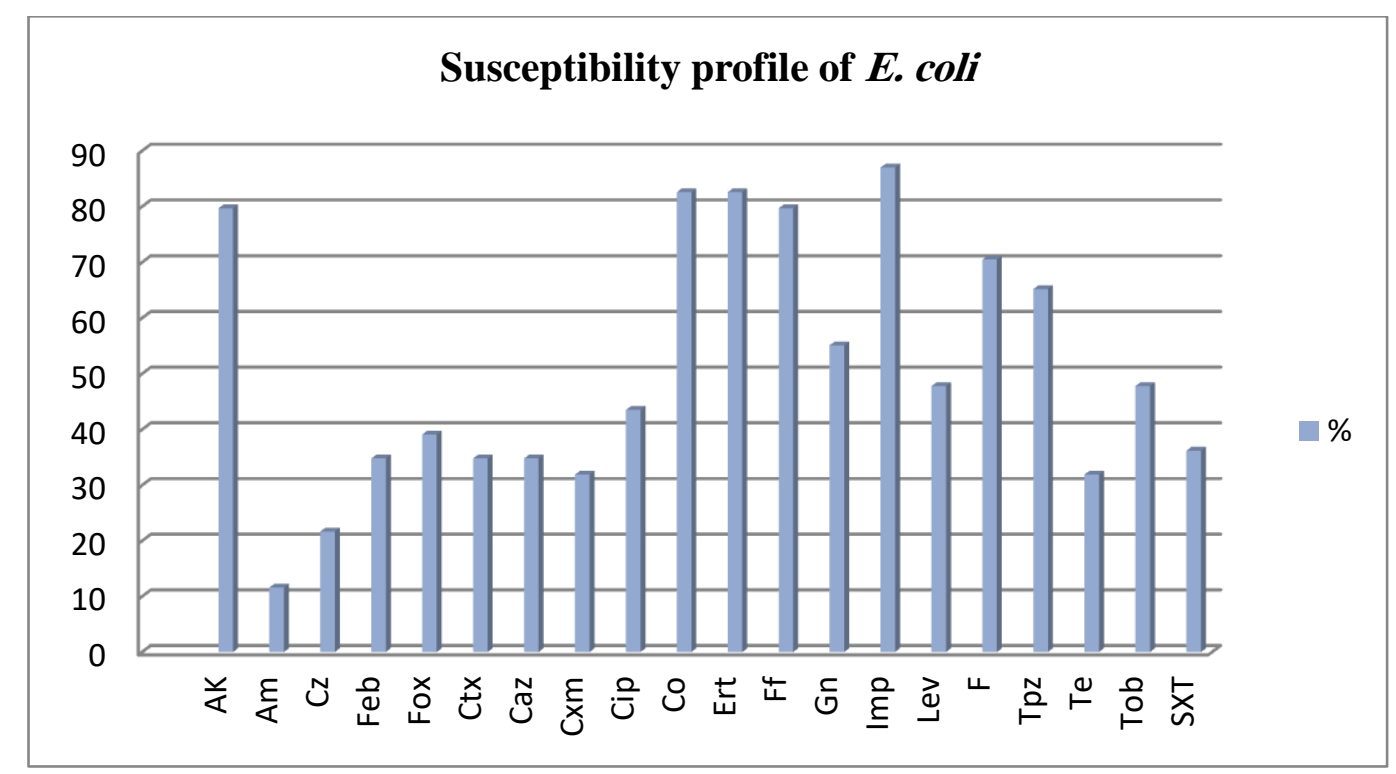

Figure 1: Susceptibility profile of $E$. coli

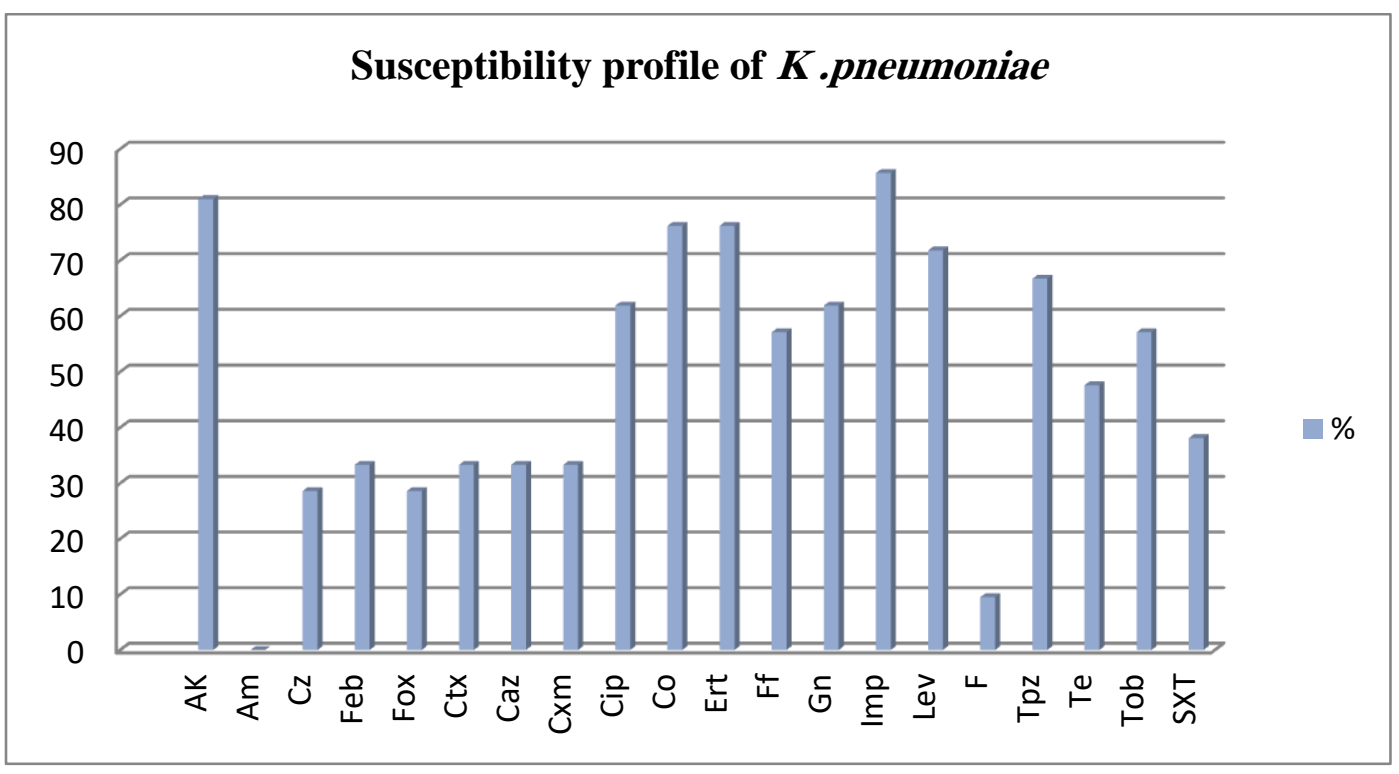

Figure2. Susceptibility profile of $K$. pneumoniae 


\section{Proceeding of the $2^{\text {nd }}$ Scientific Conference on Women's Health \\ 2-3 September 2020 - Hawler Medical University}

\section{Antibiotic susceptibility profile of $S$. aureus.}

The susceptibility profile of $S$. aureus, against various antimicrobials, is shown in Figure 2. The most isolate of S.aureus was methicillin resistance S.aureu according to the cefoxitin screen test $2(11.1 \%)$.

The test shows moderate activity against linezolid, Daptomycin, Nitrofurantoin, vancomycin, and trimethoprim -sulphamethoksasol72.2\%, 72.2\%,72.2\%, 61.1\%, 61.1\%, respectively, while the less activity was against ciprofloxacin, levofloxacin and tetracycline $16.7 \%, 22.2 \%$, $16.7 \%$ respectively.

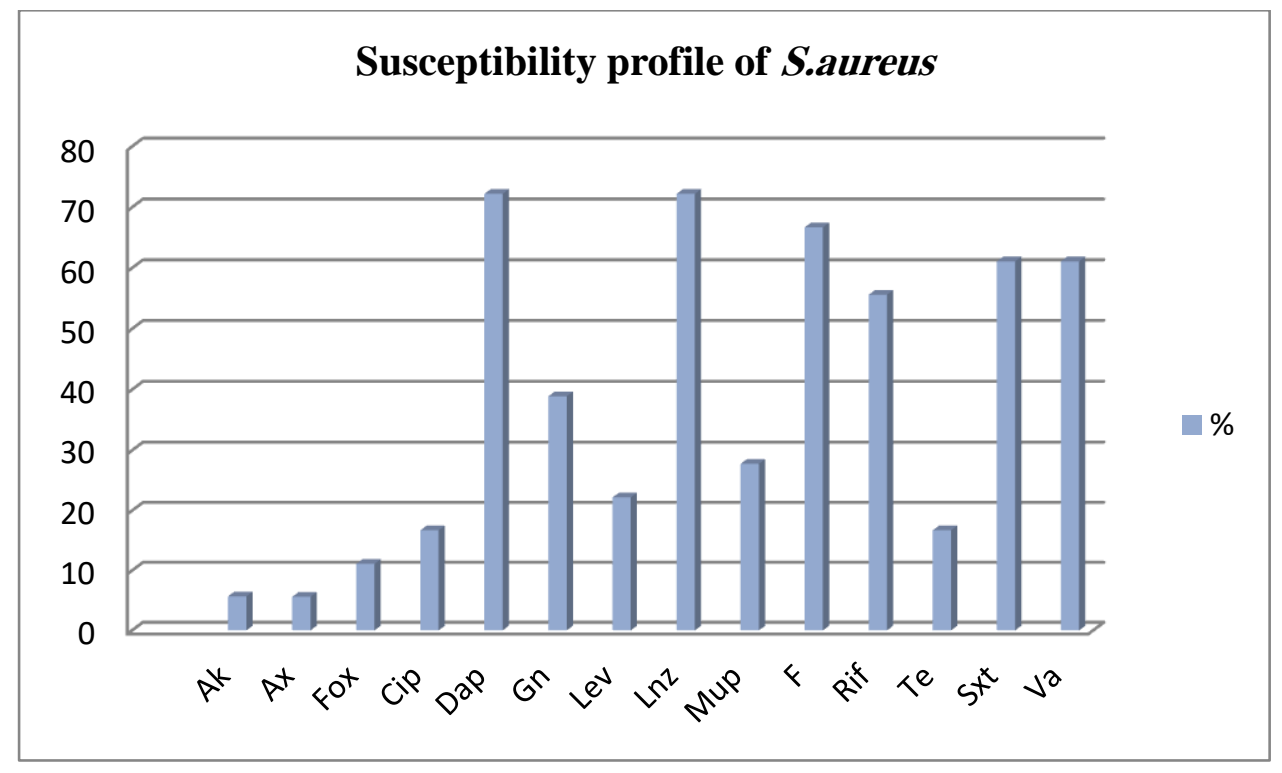

Figure3: Susceptibility profile of S.aureu

\section{DISCUSSION}

UTIs represent one of the most common diseases experienced in medical practice ${ }^{20}$. This disease affects people of all age groups, being more mutual in women. ${ }^{21-22}$ In most cases, there is a necessity to started prophylactic therapy before culture and sensitivity results are available. ${ }^{23}$ The overall isolation rate of uropathogens in this study was $29.2 \%$. This is relatively near to the results of a study performed by a former work. ${ }^{24}$ In this study, out of 298 patients who were suspected of having community-acquired (CA) - URI was attended to Bagdad's teaching hospital for a period from April 2012 to October 2012. Positive urine culture was detected in $33.6 \%$ of patients. However, our result was higher than a study performed on urine samples from 459 patients with clinical symptoms suspected to be UTI were collected from Teaching Laboratories Center in Baghdad during the study period (1st January 2009 - 1st July 2009). ${ }^{25}$ Out of 459 urine samples, 100 (21. 8\%) were showed to be 


\section{Proceeding of the $2^{\text {nd }}$ Scientific Conference on Women's Health \\ 2-3 September 2020 - Hawler Medical University}

positive urine culture. On the other hand, our result rates were lower than a study reported by Hammoudi AA ${ }^{26}$ from Al Karama teaching hospital, Baghdad. In this study, out of 280 patient samples, 162 (57. 9\%) were urine culture positive. This variation of data can be explained according to the variation of sample size, different settings ( CA-UTI-HA-UTI), and other methods to identify UTI.

Our study showed a high UTI currency in females (\%67.73) than in males (32.27\%), which correlate with other findings, which revealed that UTI frequency is greater in females than males, ${ }^{27-28}$ respectively. The high currency of UTI in females is due to close proximity of the urethral meatus to the anus, shorter urethra, sexual intercourse, and incontinence ${ }^{29-30}$.

UTIs are mostly caused by Gram-negative bacteria like E. Coli, Klebsiella spp., Proteus mirabilis (p. mirabilis), P. aeruginosa, Acinetobacter spp., Serratia spp. and Gram-positive bacteria such as Enterococcus spp. and Staphylococcus spp..In our study, like many other similar studies, E. coli $42.58 \%$ was found the most prevalent Gram-negative bacteria following by K. pneumoniae $13.5 \%$ and $S$. aureus $11.4 \%$. similar finding is shown by AlJubouri \& Mdish ${ }^{31}$ that reported E. coli and Klebsiella spp as the leading UTI pathogens, Similarily a study from Erbil Rizgary hospital was performed by Kireşçi et al. ${ }^{32}$. In this study E. coli constituted the predominant organism and was responsible for 41 (58.57\%) cases of UTI. This was followed by S. aureus 10 (14.29\%). In a recent study from Kirkuk, Iraq, Alsamarai et al. ${ }^{33}$ found that in 563 urine samples, 234 (41.6\%) were culture positive, and $E$. coli was the predominantly $(57.7 \%)$ isolated bacteria, followed by $S$. aureus $17.5 \%$, Klebsiella spp. 14.5\%, and Proteus spp. 10.3\%.

Internationally this finding is in agreement with the common knowledge about the causative agents of UTI such as that reported by Farajnia et al. ${ }^{34}$ Urine samples were collected from 5136 outpatients suspected of having a UTI. In this study, E. coli was the most mutual etiological agent of UTI $74.6 \%$, followed by Klebsiella spp. $11.7 \%$. According to data from Turkey, ${ }^{35}$ E. coli and Klebsiella spp. were major uropathogens in CA-UTI, and various Gram-positive or negatives were seen infrequently with less than 5\% ratios. Another study from Al -zarka -jordun; ${ }^{36}$ showed that more than $80 \%$ of urinary tract infections are caused by a single bacterial species E. coli followed by Klebsiella spp. 11.7\%. A study from El batman Medical Center (BMC), Tobruk- Libya, during 2003 and 2004. A total of 773 midstream urine samples from outpatients and inpatients who attended Central Lab in El Batnan Medical Center (BMC) were included. Eight different bacterial species were isolated from only $262(33.9 \%)$ E. coli strains isolated in 106 (40.4\%) of cases with UTI, Klebsiella spp. in 59 (22.5\%), S. epidermidis in 35 (13.3\%), S. aureus in 32 (12.2\%), Proteus spp.in 12 


\section{Proceeding of the $2^{\text {nd }}$ Scientific Conference on Women's Health \\ 2-3 September 2020 - Hawler Medical University}

(4.5\%), while $8(3 \%), 6(2.3 \%), 4(1.5 \%)$ of cases showed Pseudomonas, Entrococci and Corynebacteriare spectively ${ }^{37}$. In an Italian study ${ }^{38}$ collected from 61273 urine samples over a 22-month period, predominance of bacteria were with values as follows: E. Coli (67.6\%), K. pneumoniae (8.8\%), E. faecalis (6.3\%), P. Mirabilis (5.2\%), P. aeruginosa (2.5\%). In another multi-national (including Turkey) and multi-center study, a total of 1762 isolates were collected from 38 centers in 11 countries from patients with UTIs in 2009 and 2010. Enterobacteriaceae comprised $86.0 \%$ of the isolates, of which E. coli $(56.5 \%)$ and $K$. pneumoniae (13.8\%) were the two most common species. ${ }^{39}$ A retrospective analysis of the culture isolates obtained from urine samples received at the Department of Microbiology, St. John's Medical College Hospital, Bengaluru India, was performed between January 2012 and May 2012. Of the 5592 urine specimens received, 28.2\% showed significant growth. A total of 1673 identified pathogens were used in the analysis. E. coli $(54.6 \%)$ was the most common Gram-negative bacillus, followed by Klebsiella species (9.7\%) and Pseudomonas species $7.5 \% .^{40}$

The resistance rate of commonly prescribed antibiotics for UTI patients is increasing both in developing and developed countries. Resistance has emerged even to more potent antimicrobial agents. ${ }^{41}$ According to the ARMIN resistance monitoring project data in the German federal state of Lower Saxony, for instance, the resistance of E. coli to ciprofloxacin has increased from $10.3 \%$ to $14.7 \%$ in the past ten years. ${ }^{42}$ Growing resistance to cotrimoxazole and ampicillin has also been noted. ${ }^{43}$ The PubMed database was systematically searched to identify relevant studies published after 2000. Twenty-eight studies, accounting for 381,899 urine isolates from 14 African countries, met the inclusion criteria. E. coli, Klebsiella spp., and Proteus spp. were the most commonly isolated uropathogens. In this study, the highest sensitivity rate of E. coli among outpatients were for imipenem and fosfomicin, respectively $100 \%$. While cefotaksim and ciprofloxacin were the other two antibiotics with the highest activity against $E$. coli with a susceptibility rate of 92 to 99 and 68 to $91 \%$, respectively. Also the susceptibility rate of Klebsiella spp. isolates from outpatients varied from 80 to $100 \%$ for amikacin and from 53 to $100 \%$ for ciprofloxacin, while susceptibility rates were 74 to 78,97 , and $77 \%$ for ciprofloxacin, amikacin, and fosfomycin, respectively, among Klebsiella spp. isolates from inpatients or patients with hospital-acquired UTIs. $^{44}$

The current international and European clinical practice guidelines for treating acute uncomplicated UTIs and complicated UTI include nitrofurantoin, trimethoprim- 


\section{Proceeding of the $2^{\text {nd }}$ Scientific Conference on Women's Health \\ 2-3 September 2020 - Hawler Medical University}

sulfamethoxazole, fosfomycin trometamol, pivmecillinam, fluoroquinolones (ofloxacin, ciprofloxacin, and levofloxacin), and $\beta$-lactam agents (amoxicillin-clavulanate, cefdinir, cefaclor, and cefpodoxime). aminopenicillins, cephalosporins, other fluoroquinolones, and carbapenems are prescribed as cUTItreatment. ${ }^{45}$

In our study, susceptibility rate of E.coli as the main causative agent was highest against imipenem 87\%, colistin 82.6\%, amikacin 79.7\%, fosfomisin $79.7 .1 \%$, While the susceptibility rate against nitrofurointoin $70.5 \%$ trimethoprim/ sulphamethaxazol, ciprofloxacin , levofloxacin were $70.5 \%, 36.2 \%, 43.5 \%), 47.8 \%$ respectively. The lower sensitivity rate was shown against Aminopenicillin group (ampicillin 11.6\%) and Cephalosporin (Cefazolin 21.7\%, Cefepim 34.8\%, Cefoxitin 39.1\%, Cefotaxim 34.8\%, Ceftazidim 34.8\%, and Cefuroxim 31.9\%) as shown in figure 1. However, these results were in accordance with the result performed by Mahmud MA from Bagdad city ${ }^{26}$ urine samples from 459 patients with clinical symptoms suspected to be UTI were collected from Teaching Laboratories Center in Baghdad during the study period (1st January 2009 - 1st July 2009). The majority of Gram-negative bacteria showed susceptibility towards imipenem 95.3\% followed by amikacin $91.8 \%$, nitrofurantion $70.6 \%$. Ampicillin was found the least effective drug, $9.4 \%$, followed by cefoxitin, $18.8 \%$. Other tested antibiotics were effective only for less than half of Gram-negative bacterial isolates. In another study had done by Husein et al., ${ }^{25}$ from teaching hospital Iraq were included. E.coli presented a sensitivity rate to amikacin of $97.4 \%$, followed by $89.7 \%$ to imipenem, $84.6 \%$ to nitrofurantoin, $35.9 \%$ to ciprofloxacin. For ampicillin, amoxiclave, co-trimoxazole, and cephalosporin groups, E.coli showed sensitivity rate ranged from $25.6 \%$ to $35.9 \%$. Another study conducted in Erbil city and included 300 children with signs and symptoms of urinary tract infection found that most of uropathogens isolates had a very high degree of resistance to penicillin, ampicillin, sulfamethaxazole-trimethoprime, cephalothin, ceftriaxone, and cefotaxime sodium. ${ }^{46}$

In a recent study from Messalata Central Hospital, Libya, a total number of 1153 urine samples were collected from patients who attended daily to Messalata Central Hospital, Libya, in a study extended for one year. Antimicrobial susceptibility testing and isolates identification were made using Phoenix BD. The isolated uropathogens showed an increased resistance rate of $10.5 \%$ to $64.5 \%$, with an overall resistance of $28.9 \%$. Amikacin was the most effective antimicrobial followed by Imipenem and Meropenem $(0 \%, 0.6 \%$, and 2.5\% resistance, respectively); while, Cephalothin and Ampicillin were the least (80.6\% and 90.0\% resistance, respectively. ${ }^{47}$ A more recent study performed in in India by Law hale MA 59 


\section{Proceeding of the $2^{\text {nd }}$ Scientific Conference on Women's Health \\ 2-3 September 2020 - Hawler Medical University}

showed marked resistance to doxycycline, quinolones, cephalosporin's and greater sensitivity to nitrofurantoin fosfomycin. The most common isolate $E$. coli showed high resistance to commonly used empirical antibiotics like Cephalosporins 60\%, levofloxacin 52.01\%, and doxycycline $65 \%{ }^{48}$

Klebsiella spp. cause approximately $8 \%$ of healthcare-associated infections in the United States. Twenty-seven percent of healthcare-associated infections are catheter-associated urinary tract infections (UTIs) and, among facilities reporting these to the National Healthcare Safety Network (NHSN), 20\% observed that at least one Klebsiella spp. was carbapenem-resistant. ${ }^{49}$ In our study, the resistance rate of K. pneumoniaea second etiologic agent causing UTI were found highly resistant against Cephalosporin, Trimethoprim/ Sulphamethaxazol, tetrasycliin, and nitrofurontoin. In comparison, the most effective antibiotic was found to be imipenem and amikasin as shown in figure 2. These results were in accordance with the results of another researcher from Bagdad, Babylon, and Tikrit city ${ }^{24,50-}$ ${ }^{51}$. Internationally simller result were obtained from Turkey, Iran, Jordun, New Delhi. ${ }^{50-55}$

Staphylococcus aureus (SA) is a nosocomial bacterial pathogen and is a relatively uncommon cause of UTI, MRSA bacteriuria occurs among long-term care patients as well, and it is significantly associated with urinary catheterization and antibiotic use. ${ }^{56}$ Our study revealed that S.aureus were constituted $18(11.5 \%)$ of the urine sample as the third etiologic agent after E.coli and K.pneumoniae. Most of the sample were Metisilline resistant S. aureus $(89.9 \%)$, as shown in figure 3 . The izolate of MRSA revealed high resistance against amikasin, ciprofloksasin, levofloksasin, mupirocin; as shown in (figure 3) daptomisin, linezolid, synercid were the most effective antibiotic against MRSA. At the same time, vancomisin, Trimethoprim / Sulphamethoksat, Nitrofurointoin, rifampin were moderately sensitive against MRSA isolate. The sensitivity rate in our result was lower campare to the results of other researchers from Bagdad and Erbil city. ${ }^{24,26,31,46}$

Our results were also higher than a recent study from the university hospital in Cambridge, UK. In this study from 2005 through to 2014, retrospectively reviewed urine cultures recorded in a laboratory database. In total, 690 cultures were positive for SA, of which 293 (42.5\%) were methicillin-resistant. Both methicillin-sensitive Staphylococcus aureus (MSSA) and MRSA isolates were susceptible to vancomycin and nitrofurantoin. MRSA isolates demonstrated some increased resistance to trimethoprim and gentamicin and greatly increased resistance to Fluoroquinolones. ${ }^{57}$ 


\section{Proceeding of the $2^{\text {nd }}$ Scientific Conference on Women's Health 2-3 September 2020 - Hawler Medical University}

However, our results regarding (E.coli, K.pneumoniae, S.aureus) sensitivity rate were variable regionally and internationally. This variation can be explained partly due to variation of sample size, deferent setting (hospital-community), variation in age and sex group, and the most important one is a variation of method which used for detection of antibiotic sensitivity (Disk diffusion, MIC, E test). Another alarming point of this study was the low sensitivity rate of isolated uropathogens to most of the commonly used antimicrobials, including Penicillins Cephalosporins; Fluoroquinolones, and Trimethoprim - Sulphamethoksat. One of the most important factors for this situation is the massive and misuse of these antimicrobials. Besides, they are also purchased directly from the pharmacies without doctors' prescription. Self-medication is common practice with the absence of restricted rules and recommendations to guide and control infections.

\section{CONCLUSIONS}

E.coli, K.pneumoniae, S.aureus were the commonly isolated bacteria from urine samples in our regional teaching hospital. Many isolated bacterial microbes were resistant to antibiotics commonly used in clinical practices like (Penicillins, Cephalosporins, Fluoroquinolones, and Trimethoprim-Sulphamethoksat). İmipenem and amikacin were the most effective antibiotics against E.coli, and K. pneumoniae while, daptomisin, linezolid, and synercid were the most effective antibiotics against S.aureus.

\section{REFERENCES}

1- Singhal A, Sharma R, Jain M, Vyas L. Hospital and Community Isolates of Uropathogens and their Antibiotic Sensitivity Pattern from a Tertiary Care Hospital in North West India. Ann Med Health Sci Res 2014 ; 4(1):51-6.

2- Dromigny JA, Nabeth P, Juergens BA, Perrier-Gross-Claude JD. Risk factors for antibiotic resistance Escherichia coli urinary tract infection in Dakar; Senegal. J Antimicrob Chemother 2005; 56: 236-39.

3- Bronsema, DA, Adams JR, Pallares R. Secular trends in rates and etiology of nosocmial urinary tract infections at auniversity hospital; J Urol 1995; 150: 414- 16.

4- Kim G, Joseph T, Urinary Tract Infection Update. AJOCM 2012; 1 9(2).

5- Moroh et al. Diversity and antibiotic resistance of uropathogenic bacteria from Abidjan. Afr J Urol 2014; 20(1):18-24. 
6- Carlet J, Jarlier V, Harbarth S, Voss A, Goossens H, Pittet D. Ready for a world without antibiotics? The Pensières Antibiotic Resistance Call to Action. Antimicrob Resist Infect Control 2012;1(11) doi: 10.1186/2047-2994-1-11

7- Kang C, Song J. Antimicrobial Resistance in Asia: Current Epidemiology and Clinical Implications. Clinical Implications. Infect Chemother. 2013;45(1):22-31. doi: 10.3947/ic.2013.45.1.22.

8- Resistance Global Report on WHO Antimicrobial Surveillance [Online], 2014. Available from: http://www.thehealthwell.info/ node/763364.

9- Wagenlehner FM, Niemetz AH, Weidner W, Naber KG. Spectrum and antibiotic resistance of uropathogens from hospitalised patients with urinary tract infections: 1994-2005. Int J Antimicrob Agents 2008;31 (1): 25-34.

10- Baraboutis et al. Primary Staphylococcus aureus urinary tract infection: the role of undetected hematogenous seeding of the urinary tract. Eur J Clin Microbiol Infect Dis 2010; 29:1095-101. doi:10.1007/s10096-010-0967-2.

11-Choi et al. The clinical significance of concurrent Staphylococcus aureus bacteriuria in patients with S. aureus bacteremia. J Inf Secur 2009; 59: 37-41.

12- Todar K. Bacterial resistance to antibiotics. Todar's online textbook of bacteriology. 2011.

13- Diller et al. Evidence for cost reduction based on pre-admission MRSA screening in general surgery. Int J Hyg Environ Health 2008; 211:205-12.

14- Lunacek A, Koenig U, Mrstik C, Radmayr C, Horninger W, Plas E. Unexpected Multidrug Resistance of Methicillin-Resistant Staphylococcus aureus in Urine Samples: A Single-Center Study. Korean J Urol 2014; 55(5):349-53.

15-Orrett FA, Land M. Methicillin-resistant Staphylococcus aureus prevalence: current susceptibility patterns in Trinidad. BMC Infect Dis 2006; 6:83.

16- Hiramatsu K, Katayama Y, Yuzawa H, Ito T. Molecular genetics of methicillin resistant Staphylococcus aureus. Int J Med Microbiol 2002; 292: 67- 74.

17- Aires de Sousa M, de Lencastre H. Bridges from hospitals to the laboratory: genetic portraits of methicillin-resistant Staphylococcus aureus clones. FEMS Immunol Med Microbiol 2004; 40:101- 11.

18- Koneman's Color Atlas and Textbook of Diagnostic Microbiology, 6th Edition By Washington Winn Jr. Stephen Allen, William Janda, Elmer Koneman, Gary Procop, 
Proceeding of the $2^{\text {nd }}$ Scientific Conference on Women's Health

2-3 September 2020 - Hawler Medical University

Paul Schreckenberger, and Gail Woods. Pp: 1736. Lippincott Williams \& Wilkins, Philadelphia, 2005.

19- Clinical and Laboratory Standard Institute. Performance standards for antimicrobial susceptibility testing; 20th ed. Wayne, Pennsylvania, USA. CLSI 2014. M100-S20.

20- Eliana BMG, Berezin EN, Nigro S, Nataly AS, Benini V, Toporovski J. Antibiotic resistance patterns of pediatric community-acquired urinary tract infections. Braz $\mathbf{J}$ Infect Dis 2008; 12(4):321-323.

21-Enayat K, Fariba F, Bahram N. Asymptomatic bacteriuria among pregnant women referred to outpatient clinics in Sanandaj, Iran. Int Braz J Urol 2008; 34:699-707.

22-Rock W, Colodner R, Chazan B, Elias M, Raz R. Ten years surveillance of antimicrobial susceptibility of community acquired Escherichia coli and other uropathogens in Northern Israel. Israel Med Assoc J 2007; 9:803-5.

23-Gupta K: Emerging antibiotic resistance in urinary tract pathogens. Infect Dis Clin North Am 2003;17:243-59.

24- Hussein NS. Clinical, Etiology and Antibiotic Susceptibility Profiles of CommunityAcquired Urinary Tract Infection in a Baghdad Hospital. Med Surg Urol 2014; 3:136.

25- Mahmood MA. Prevalence and Antimicrobial Susceptibility of Pathogens in Urinary Tract Infections. Journal of Al-Nahrain Unv 2011;14 (4) :146-52.

26- Hammoudi AA, Urinary Tract Infection of adults in Baghdad City. Int J Curr Microbiol App Sci 2013; 2(10):1-6.

27- Mansour A, Manizeh M, Zohreh P. Study of bacteria isolated from urinary urinary tract infections and determination of their susceptibility to antibiotics. Jundishapur $\mathrm{J}$ Microbiol. 2009; 3:118-20.

28- Rajalakshmi V, Amsaveni V, Antibiotic susceptibility of bacterial pathogens isolated from diabetic patients. Intern J of Microbiol Res 2012; 3(1) :30-32.

29- Oladeinde BH, Omoregie R, Olley M, Anunibe JA. Urinary tract infection in a rural community of Nigeria. N Am J Med Sci 2011; 3(2):75-7. doi: 10.4297/najms.2011.375. PubMed PMID: 22540069; PubMed Central PMCID: PMC3336890.

30-Dimitrov T S, Udo E E, Emara M, Awni F, Passadilla R, Etiology and Antibiotic Susceptibility Patterns of Community-Acquired Urinary Tract Infections in a Kuwait Hospital. Med Princ Pract 2004;13:334-39.

31-Al-Jebouri MM, Mdish SA, Antibiotic Resistance Pattern of Bacteria Isolated from Patients of Urinary Tract Infections in Iraq.Open Journal of Urology 2013 ; 3: 124- 
Proceeding of the $2^{\text {nd }}$ Scientific Conference on Women's Health

2-3 September 2020 - Hawler Medical University

31.

32- Kireçci E, Sleman DM, Ahmed DY, Rahman DB and Yazdee FS, Identification of the bacterial types that cause urinary tract infection and antimicrobial susceptibility in Erbil, Iraq. SJMR 2015; 3(1): 011 - 014

33- Alsamarai AGM, Ali S. Urinary tract infection in female in Kirkuk city, Iraq: causative agents and antibiogram. World J Pharm Pharmaceut Sci 2016; 5(6):261-73.

34- Farajnia S, AlikhanivMU, Ghotaslou R, Naghili B, and Nakhlband A. A causative agent and antimicrobial susceptibilities of urinary tract infection in the North west of Iran. IJID 2008; 13:140-4.

35- Coşkun Ö, Erdem H, Avcı A. Management of community-acquired acute bacterial cystitis in Turkey. Turk J Med Sci 2011; 41(1): 149-157.

36- Alsohaili SA, Alharahsheh MH, Almshagbeh MA, Alkhawaldeh RA, ALkhawaldeh WM. Bacterial pathogen in urinary tract infection and antibiotic resistance patteern in zaraqa -Jordan. ESJ 2015; 11(12).

37- Dabobash MD, Attla MF, Elgarba M, Menshawy ASA . Antibiogram Sensitivity in Urinary Tract Infections (UTI)at El Batnan Medical Center- Tobruk - Libya. UNOAJ 2017;4(3): 00129. DOI: 10.15406/unoaj.2017.04.00129.

38- Magliano et al. Gender and age-dependent etiology of community-acquired urinary tract infections. SWJ 2012; http://dx.doi.org/10.1100/2012/349597.

39- Lu et al. Epidemiology and antimicrobial susceptibility profiles of Gram-negative bacteria causing urinary tract infections in the Asia-Pacific region: 2009-2010 results from the Study for Monitoring Antimicrobial Resistance Trends (SMART). Int J Antimicrob Agents. 2012;40(Suppl):37-43. doi:10.1016/S0924-8579(12)70008-0

40- Kalal BS, Nagaraj S. Urinary tract infections: a retrospective, descriptive study of causative organisms and antimicrobial pattern of samples received for culture, from a tertiary care setting. Germs 2016; 6(4):132-138. doi:10.11599/germs.2016.1100.

41- Thiraviam M, Yadesa D, Adugna T. Antibiotic resistant pattern of urinary tract infection causing Escherichia coli isolated from diabetic mellitus and non-diabetic mellitus patients with special reference to Rifampicin resistance. Int J Curr Microbiol App Sci 2014; 3:668-74.

42- Niersächisches, Gesundheitsamt:ARMIN.www.nlga.niedersachsen.de/infektionsschutz /armin_resistenzentwicklung/armin_inter aktiv 2017.

43-Zwirner M, Bialek R, Roth T, et al. Local resistance profile of bacterial isolates in uncomplicated urinary tract infections (LOREstudy). Kongress abstract DGHM 2016. 
44- Tansarli GS, Athanasiou S, Falagas ME. Evaluation of antimicrobial susceptibility of Enterobacteriaceae causing urinary tract infections in Africa. Antimicrob Agents Chemother 2013; 57: 3628-39.

45- Tan CW, Chlebicki MP, Urinary tract infections in adults. Singapore Med J 2016; 57(9): 485 - 90.

46- Saeed HC. AL-Otraqchi K, Mansoor BK. Prevalence of urinary tract infections and antibiotics susceptibility pattern among infants and young children in Erbil city. Zanco J Med Sci 2015;19:915-22.

47- Muhamed MA, Alnour TM, Sharkufor OM, Aburass MM, Prevalence and antimicrobial resistance pattern of bacterial strains isolated from patients with urinary tract infection in Messalata Central Hospital, Libya. Asian Pac J Trop Med 2016, $9(8): 771-6$.

48- Lawhalel MA, Naikward R. Recent pattern of drug sensitivity of most commonly isolated uropathogens from Central India. Int J Res Med Sci 2017;5(8):3631-36.

49- Brizendine KD, Richter SS, Cober ED, van Duin D. Carbapenem resistant Klebsiella pneumoniae urinary tract infection following solid organ transplantation. Antimicrob Agents Chemother 2015; 59: 553-57.

50- Alhamdany MA. Antibiotic susceptibility of bacteria isolated from patients with diabetes mellitus and recurrent urinary tract infections in Babylon Province, Iraq. Med J Babylon 2018; 15:63-8.

51- Alsamarai MA, Rahman IA, Abdu-aziz MM, Urinary Tract Infection In Iraq: Evaluation Of Early Detection Methods And Etiology. Int J Pharm Pharm Sci. 2016; $5(7): 81-194$.

52- Gülcan A, Aslantürk A, Gülcan E, The Microorganisms Isolated From Urine Culture and Their in Vitro Antibiotic Susceptibility. Abant Med J 2012; 1(3 ):129-35.

53- Mirza IA, Ikram A, Afzal A, Ali S, Hussain A, Fayyaz M, Ghafoor T. Antimicrobial Susceptibility Pattern of Bacteria Isolated from Patients with Urinary Tract Infection. J Coll Physicians Surg Pak 2014 ; 24(11):840-4.

54- Hayajneh et al. Susceptibility trends and molecular characterization of Gram-negative bacilli associated with urinary tract and intra-abdominal infections in Jordan and Lebanon: SMART 2011-2013. Int J Infect Dis 2015; 35:56-61.

55- Rizwan M, Akhtar M, Najmi AK, Singh K; Escherichia coli and Klebsiella pneumoniae Sensitivity Resistance Pattern Towards Antimicrobial Agents in Primary 
Proceeding of the $2^{\text {nd }}$ Scientific Conference on Women's Health 2-3 September 2020 - Hawler Medical University

and Simple Urinary Tract Infection Patients Visiting University Hospital of Jamia Hamdard New Delhi, Drug Res (Stuttg) 2018; 68(7):415-20.

56- Garner JS, Jarvis WR, Emori TG, HoranTC, Hughes JM.CDC definitions for nosocomial infections. Am J Infect Control 1988; 16:128-40.

57- Toner et al. Staphylococcus aureus urinary tract bacteriuria: single-institutional antibiotic susceptibility trends over a decade. Bladder 2017;4(3):30. 